\title{
Effectiveness of Turmeric Mouthwash and Sodium Bicarbonate Mouthwash to Reduce Oral Mucositis among Patient Undergoing Radiation Therapy
}

\author{
Mr. P. Ruban David ${ }^{*}$, Ms. K. Timple Shree ${ }^{2}$ \\ ${ }^{1}$ Assistant Professor, Department of Medical Surgical Nursing, Saveetha College of Nursing, Saveetha Institute of Medical and Technical Sciences, \\ No.162, Poonamallee High Rd, Velappanchavadi, Chennai, Tamil Nadu 600077, India \\ ${ }^{2}$ B.Sc Nursing IV year, Saveetha College of Nursing, Saveetha Institute of Medical and Technical Sciences, No.162, Poonamallee High Rd, \\ Velappanchavadi, Chennai, Tamil Nadu 600077, India
}

DOI: 10.36348/SIJTCM.2019.v02i07.001 | | Received: 09.09.2019| Accepted: 16.09.2019| Published: 30.09 .2019

*Corresponding author: Mr. P. Ruban David

\section{Abstract}

Objectives: To assess the level of oral Mucositis among patient undergoing radiation therapy, to assess the effectiveness of turmeric mouthwash and sodium bicarbonate mouthwash among oral Mucositis patients, to compare the effectiveness of turmeric mouthwash and sodium bicarbonate mouthwash among oral Mucositis patients and to associate the effectiveness of turmeric mouthwash and sodium bicarbonate mouthwash among oral Mucositis patients undergoing radiation therapy with selected demographic and clinical variables. Methodology: Quantitative approach-True experimental comparative design was adopted. Sample size was 60 (30 samples in group I and 30 samples in group II), assigned by simple random sampling technique method was used. National Cancer Institute-Common Toxicity CriteriaOral Mucositis grading scale was used to measure the pre test of oral Mucositis. The interventions was administration of turmeric mouth wash to group I and sodium bicarbonate mouthwash to group II for 1 minutes for 2 times a day (11 am,7pm) for 1 week for each groups. Post test was conducted for both groups on second week by using the same tool. Findings: By using unpaired t-test the calculated ' $t$ ' value for experimental group $\mathrm{I}$ is $\mathrm{t}=6.38$ which is found significant at $<0.005$ level and the ' $t$ ' value for the experimental group II is $t=0.88$. Conclusion: The finding proved that turmeric mouthwash is more effective than sodium bicarbonate mouthwash to reduce the level of oral Mucositis.

Keywords: Effectiveness, Oral Mucositis, Turmeric Mouthwash, Sodium Bicarbonate Mouthwash.

Copyright @ 2019: This is an open-access article distributed under the terms of the Creative Commons Attribution license which permits unrestricted use, distribution, and reproduction in any medium for non-commercial use (NonCommercial, or CC-BY-NC) provided the original author and source are credited.

\section{INTRODUCTION}

Mucositis is the painful inflammation and ulceration of the mucosa membranes, usually as a side effect of chemotherapy and radiation therapy for cancers [1]. Mucositis may limit the patient's ability to tolerate chemotherapy $(\mathrm{CT})$ or radiation therapy (RT), and nutritional status is compromised. It may drastically affect cancer treatment as well as the patient's quality of life. The incidence and severity of Mucositis will vary from patient to patient and from treatment to treatment. It is estimated that there $40 \%$ incidence of Mucositis in patients treated with standard chemotherapy and this will not only increase with the number of treatment of cycles but also several problems, including pain, nutritional problems as a result of inability to eat, and increased risk of infection due to open sores in the mucosa. It has a significant effect on the patient's quality of life and can be doselimiting [2].
A study was conducted in the department of dental medicine, Winthrop university hospital New York, and it stated that, the oral mucosa is a common site for collateral damage of cancer therapies including radiation, cytotoxic medications, and newer targeted therapies. Ulcerative oral Mucositis is typically painful and affects oral functions including speech, and oral intake of food and medications, thus impacting the quality of life. Prevention of oral Mucositis is an ongoing quest currently with relatively few answers. Oral Mucositis not only has a dramatic impact on the patient's quality of life, but it also can adversely influence the administration of an optimal antineoplastic treatment [3].

Turmeric is one of nature's most powerful healers. Turmeric has been used for over 2500 years in India. Research conducted at the University of 
Michigan, by Ayyaluswamy Ramamurthy in 2009, showed that curcumin in turmeric helps regulate cells by inserting itself into the cell membrane and interfering with molecular pathways that lead susceptible to infections and even to cancer [4]. The active ingredient in turmeric is curcumin. Curcumin is often recommended to protect healthy cells from harmful effect of radiation and chemotherapy, without reducing the effectiveness of these treatments [5]. The effects of a sodium bicarbonate mouthwash solution in thought to aid in the formation of granulation tissue and to promote healing. Sodium bicarbonate mouthwash solution is safe and economical and has been used in cancer patients. Sodium bicarbonate mouthwash solution gargles cleanses the wound, reduces swelling and can decrease pain [6].

\section{MATERIALS AND METHODS}

Prior permission was obtained from the ethical committee of the Institutional Research Board (IRB). In order to accomplish the main objectives of determining the effectiveness of turmeric or sodium bicarbonate mouthwash on treatment, Quantitative research approach was adopted. The research design used in this study is true-experimental comparative design. This study was conducted in radiation department at selected hospital in Chennai. A Simple random sampling technique was used. The subjects for this study consisted of 60 patients, 30 in experimental group I receiving turmeric mouthwash (Mouthwash solution prepared by mixing 5 gms of turmeric powder with 50 $\mathrm{ml}$ of water. Each time a $50 \mathrm{ml}$ of freshly prepared turmeric mouthwash solution will be administrated and 30 in group II receiving $\mathrm{g}$ sodium bicarbonate mouthwash (one teaspoon $1.5 \mathrm{Gms}$ of sodium bicarbonate powder with $50 \mathrm{ml}$ of water).each time a 50 $\mathrm{ml}$ of freshly prepared sodium bicarbonate mouthwash solution will be administrated. Semi-structure interview questionnaire which consists of demographic variables (age, gender, marital status, educational status, occupation, family monthly income) and clinical variables (stages of cancer, duration of cancer, BMI value, frequency of taking oral hygiene, life styles, habits, fractional dose of radiation therapy per day, family history of cancer). The second tool for assessment of level of oral Mucositis by using National Cancer Institute-Common toxicity criteria- Oral Mucositis grading scale. It is a standardized tool.

\section{RESULT}

The data was tabulated, analyzed and interpreted using the descriptive and inferential statistics.

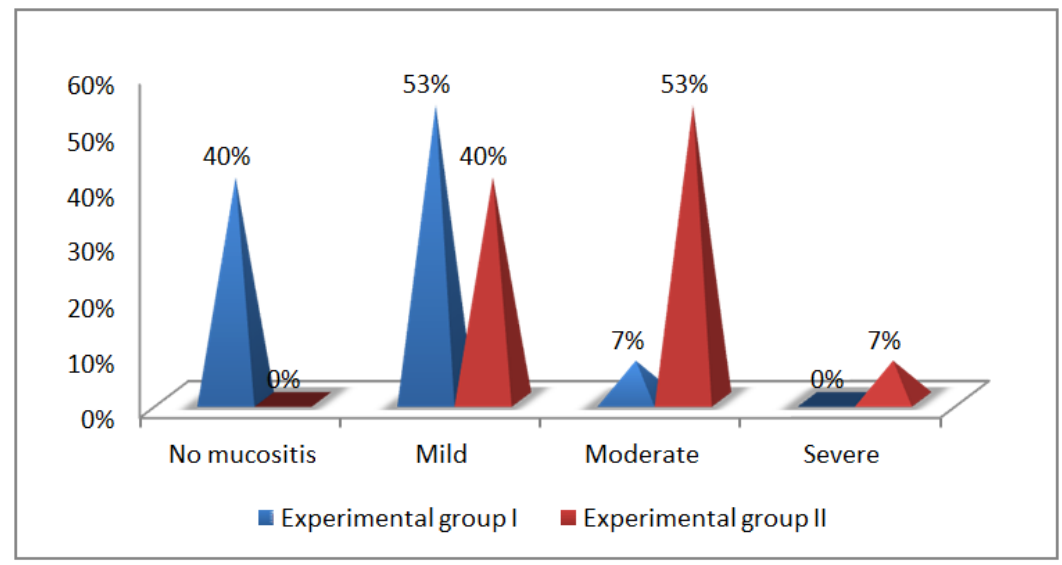

Fig-1: Total percentage of post-test score of oral Mucositis among patient undergoing radiation therapy in experimental group I and experimental group II

In experimental group I the score of oral Mucositis in No Mucositis is $12(40 \%)$, Mild 16(53\%), Moderate 2(7\%), Severe 0(0\%). In experimental group
II was No Mucositis is $0(0 \%)$, Mild $12(40 \%)$, Moderate $16(53 \%)$, Severe $2(7 \%)$.

Table-1: Mean, SD and ' $t$ ' value of oral Mucositis among patient undergoing radiation therapy in experimental group I and group II $\mathrm{N}=(\mathbf{3 0}+\mathbf{3 0}) 60$

\begin{tabular}{|l|l|l|l|l|l|}
\hline S. no & Groups & Mean SD & t value & 'p' value \\
\hline 1 & Experimental group I & 0.66 & 0.605 & 6.38 & 0.001 \\
\hline 2 & Experimental group II & 1.66 & 1.667 & 0.88 & 0.378 \\
\hline
\end{tabular}

The Table-1 reveals that experimental group I and experimental group II mean were 0.66 and 1.66. The obtained calculated $\mathrm{t}$ value was 6.38 which is greater than the table value at $\mathrm{p}<0.005$ level of significance.

\section{DISCUSSION}

In this study the level of Oral Mucositis among patients undergoing Radiation therapy were measured by NCI-CTC-Radiation induced oral Mucositis grading scale. The present study reveals that pre test score of 
NCI-CTC-Radiation induced oral Mucositis grading scale on Oral Mucositis were 18(60\%) participants were in severe Oral Mucositis, $11(37 \%)$ participants were in moderate level of Oral Mucositis, 1(3\%) participants were in mild level of oral Mucositis in each experimental group I and experimental group II.

This study was supported by Luiz Evaristo Ricci Volpato et al., conducted a study on Oral Mucositis. Nearly all (90\% to $97 \%$ ) patients receiving radiotherapy in the head and neck will develop some degree of Mucositis. Of these Patients treated with radiotherapy with or without chemotherapy, 34\% to $43 \%$ will present severe Mucositis. As a result, the patient's quality of life is affected, hospital admittance rates are higher, the use of total parenteral nutrition is increased and interruption of treatment is more frequent, all of which compromise tumor control. Mucositis causes 9\% to $19 \%$ of chemotherapy and radiotherapy interruption [7].

This study was supported by Trotti A Bellm L A et al., conducted study to determine the frequency of Mucositis and associated outcomes in patients receiving radiotherapy (RT) for head and neck cancer through a systematic review. According to the study protocol, databases were searched for randomized clinical 101trials (English only, 1996-1999) of patients with head and neck cancer receiving RT were $16 \%$ overall with or without chemotherapy that reported one or more outcomes of interest. Thirty three studies $(n=6181$ patients) met inclusion criteria. Mucositis was defined using a variety of scoring systems. The mean incidence was $80 \%$. Over one-half of patients $(56 \%)$ who received altered fractionation RT (RT-AF) experienced severe Mucositis (grades 3-4) compared to 34\% of patients who received conventional RT. Rates of hospitalization due to Mucositis, reported in three studies $(n=700)$, and $32 \%$ for RTAF patients. Eleven percent of patients had RT regimens interrupted or modified because of Mucositis in five studies $(n=1267)$ reporting this outcome [8].

The mean post test score of NCI-CTCRadiation induced oral Mucositis was 0.66 among patients with varying grade of Radiation induced oral Mucositis in Experimental group I. After administering turmeric mouth wash its level was significantly lower than their mean pre test score of NCI-CTC-Radiation induced oral mucositis 2.56. This findings reveals that turmeric mouth wash is very effective in the healing of Radiation induced 1oral Mucositis In the Experimental group II, the mean post test score of oral Mucositis has considerably reduced from 2.7 to 1.66 . This findings reveals that Sodium bicarbonate is also effective in the healing of oral Mucositis.

The study has been supported by Elsevier 2011 conducted a Randomized clinical trial. In this study comparison made between patient preference for a new supersaturated calcium phosphate oral rinse, Neutra Sal to our historical rates for patients using standard salt and soda rinses. 35 patients were evaluated all receiving radiation therapy, 12 of them received both chemotherapy and radiation therapy concurrently, for confirmed Squamous cell or adeno carcinoma cancer. They were evaluated utilizing Neutra Sal against the standard of care salt and soda rinses. Historical degrees of the side effects using the standard of care option and the NeutraSal.

Patients were evaluated weekly during treatment and approximately 4-6 weeks for the acute toxicities and subsequent follow up every 4-8 week for 9 months post treatment .Among the 35 patients evaluated, it was found that the oral toxicities was found that the oral toxicities associated with radiation therapy were significantly lower than Neutra Sal rinses was utilized. The findings of this study revealed that routine use of standard salt and soda oral rinse by patients undergoing head and neck radiation significantly reduced the severity of acute mucosal toxicity and compares favorably to outcomes with and soda rinses [9].

In Experimental group I, the mean value of pre test is 2.56 and the mean value of post test is 0.66 and the ' $t$ ' value for experimental group $I$ is ' $t$ ' $=6.38$ which is found significant at $\mathrm{P}<0.005$ level. In Experimental group II, the mean value of pre test is 2.7 and the mean value of post test is 1.66 and the ' $t$ ' value for experimental group II is ' $t$ ' $=0.88$ which is also found significant at $\mathrm{P}<0.005$ level. This findings depicts that, Turmeric mouth wash is more effective than Sodium bicarbonate mouth wash on Oral Mucositis.

This study was supported by Parulekar et al., have estimated that chemotherapy-induced Mucositis varies from 40 to $76 \%$ in patients treated respectively with standard and high-dose chemotherapy. Nearly all (90\% to $97 \%$ ) patients receiving radiotherapy in the head and neck will develop some degree of Mucositis. Of these patients treated with radiotherapy with or without chemotherapy, $34 \%$ to $43 \%$ will present severe mucositis [10].

\section{ACKNOWLEDGMENT}

The authors are thankful to Prof. Dr. S. KalaBarathi, Principal of Saveetha College of Nursing, SIMATS. The authors also wish cordial to Mr. Ruban David, Associate Professor, Saveetha College of Nursing, SIMATS for their encouragement, valuable suggestions, support and advice given throughout the study.

\section{REFERENCE}

1. National Cancer Institute. (2005). Oral Complications of Chemotherapy and Head/Neck Radiation.

2. Naidu, M. U. R., Ramana, G. V., Rani, P. U., 
Mohan, I. K., Suman, A., \& Roy, P. (2004). Chemotherapy-induced and/or radiation therapyinduced oral mucositis-complicating the treatment of cancer. Neoplasia (New York, $N Y), 6(5), 423-431$.

3. Barasch, A., \& Epstein, J. B. (2011). Management of Cancer induced oral Mucositis. Dermatol Ther, 1110-20.

4. Grimes, M. (2010). New research continue to support health benefit of turmeric and Curcumin. Available

at: www.naturalnews.com/029525_turmeric_curcumi n.htmt.

5. Debjit Bhowmik, C., Kumar, K. S., Chandira, M., \& Jayakar, B. (2009). Turmeric: a herbal and traditional medicine. Archives of applied science research, 1(2), 86-108.

6. Choi, S. E., \& Kim, H. S. (2012). Sodium bicarbonate solution versus chlorhexidine mouthwash in oral care of acute leukemia patients undergoing induction chemotherapy: a randomized controlled trial. Asian Nursing Research, 6(2), 60-66.

7. Symonds, R. P. (1998). Treatment-induced mucositis: an old problem with new remedies. British journal of cancer, 77(10), 1689.

8. Trotti, A., Bellm, L. A., Epstein, J. B., Frame, D., Fuchs, H. J., Gwede, C. K., ... \& Zilberberg, M. D. (2003). Mucositis incidence, severity and associated outcomes in patients with head and neck cancer receiving radiotherapy with or without chemotherapy: a systematic literature review. Radiotherapy and oncology, 66(3), 253262.

9. Markiewicz, M., Dzierzak-Mietla, M., Frankiewicz, A., Zielinska, P., Koclega, A., Kruszelnicka, M., \& Kyrcz-Krzemien, S. (2012). Treating oral mucositis with a supersaturated calcium phosphate rinse: comparison with control in patients undergoing allogeneic hematopoietic stem cell transplantation. Supportive Care in Cancer, 20(9), 2223-2229.

10. Köstler, W. J., Hejna, M., Wenzel, C., \& Zielinski, C. C. (2001). Oral mucositis complicating chemotherapy and/or radiotherapy: options for prevention and treatment. CA: a cancer journal for clinicians, 51(5), 290-315. 